\title{
POR UNA REHABILITACIÓN DEL ESTRUCTURALISMO
}

\author{
Mar LLERA LLORENTE \\ Universidad de Sevilla \\ mllera@us.es
}

Resumen: Actualmente es frecuente denostar el estructuralismo como una escuela académica obsoleta, cientificista e inmanentista. Una mirada más profunda permitiría rehabilitarlo, revelando su rica complejidad y su proximidad a la Cibernética y a la Teoría de Sistemas.

Abstract: According to the currently most common interpretation Structuralism is an obsolete intellectual school, obsessed by scientific rigour and biased by formal immanence. However, a deeper insight reveals its complexity and its inner controversy, challenging the canonical outlook while approaching Cybernetics and System Theory.

Palabras clave: Estructuralismo. Cibernética. Complejidad. Sistema.

Key words: Structuralism. Cybernetics. Complexity. System. 
El mercado de las ideas - como cualquier otro- necesita renovar periódicamente su oferta para atraer clientes. En la Academia esto significa publicar cada cierto tiempo un nuevo índice de autores y teorías proscritas, de todo lo que se considera «superado» por las nuevas investigaciones. Al leerlo nos suele sorprender la torpeza de nuestros antecesores, ilustres - sin embargo- porque un día nosotros mismos construimos su prestigio.

Muchas defenestradas celebridades del siglo Xx pertenecieron al Estructuralismo, hoy descrito como una corriente obsoleta, lastrada por el prurito positivista, obsesionada por los códigos, alejada de la vida real y la pragmática comunicativa, desconocedora del devenir temporal, recluida en la clausura de su inmanentismo formalista.

Sin embargo, ninguna lectura medianamente abierta de su legado podría soportar tal denigración. Hemos simplificado - e incluso ridiculizado- el pensamiento de los quizá mal denominados «estructuralistas» para vender mejor nuestra producción académica: el Posestructuralismo y el Posmodernismo, la Postsemiótica, las sucesivas teorías de sistemas... Pero no hemos advertido que así cometíamos el mismo error que criticábamos: encerramos el Estructuralismo dentro de los límites de una interpretación fija, conculcando nuestros principios acerca de la apertura hermenéutica, lo conjugamos en singular para exaltar incoherentemente nuestro pluralismo.

Ahora bien, Saussure y sus discípulos desmienten nuestra caricatura: el Cours de Linguistique Générale (1916) ni siquiera utiliza el concepto de «estructura». Entre los seguidores del profesor ginebrino prestaremos atención a dos opciones extremas y antagónicas: la deriva cientificista más ortodoxa —en la glosemática de Hjelmslev \& Uldall—y la disidencia —en las reflexiones estéticas de Mukarovsky-.

No queremos olvidar que ya en los años 60-70 se discutía sobre la auténtica existencia del Estructuralismo y la legitimidad de esa designación. Tampoco queremos perder de vista que el Posestructuralismo es el fruto en que se rompe y deshace la semilla estructuralista, sólo para manifestar su verdadera potencia y engendrarse de nuevo. Por otra parte, los últimos desarrollos de la Cibernética y la Teoría General de Sistemas — donde se aprecia el legado de Norbert Wiener, Claude Shannon y Gregory Bateson, entre muchos otros - no son ajenos al mismo paradigma.

Así pues, urge rehabilitar el Estructuralismo en toda la riqueza de su potencial heurístico y sus implicaciones en diversos dominios, no para anquilosarnos en el pasado, sino para hacer justicia a sus promotores y ser cohe- 
rentes con nuestros propios presupuestos metodológicos acerca de la apertura y el pluralismo interpretativos.

\section{QUÉ ES EL ESTRUCTURALISMO}

Habitualmente el Estructuralismo se caracteriza como una corriente - escuela, método, moda intelectual o incluso ideología- que desarrolla las sugerencias de Saussure en torno a la Semiología en una línea científico-positivista. De ella procede la perspectiva sincrónica e inmanentista que prima la organización formal - la estructura - sobre los materiales significantes y su dinamismo.

Pero lo cierto es que este relato académico es una tosca simplificación: oculta las controversias y diferencias entre los «estructuralistas», su polisemia e incluso equivocidad bajo una estrecha categorización, artificialmente construida ${ }^{1}$.

El Profesor ginebrino había definido la Semiología como «une science qui étudie la vie des signes au sein de la vie sociale» (Saussure, 1916/1972: 34). Sin embargo, la comunidad intelectual pronto situó el Estructuralismo en los antípodas de la Pragmática y ofreció una interpretación deliberadamente castrada del Cours de Linguistique Générale, que había sido publicado en 1916, a partir de notas tomadas por sus estudiantes en los tres cursos impartidos entre 1906 y 1911.

No se tuvo suficientemente en cuenta que Bally y Sechehaye, responsables de la edición más célebre del Cours, habían advertido con lucidez y modestia en el prefacio: «Nous sentons toute la responsabilité que nous assumons vis-à-vis de la critique, vis-à-vis de l'auteur lui-même, qui n'aurait peut-être autorisé la publication de ces pages. (...) La critique saura t-elle distinguer entre le maître et ses interprètes?» (Bally \& Sechehaye, en Saussure, 1916/1972: 11).

Este hecho explica el carácter embrionario, incompleto, a veces incoherente y siempre polisémico del legado saussureano. De hecho, los «estructuralistas» no cultivaron una sola disciplina, sino muchas y muy variadas:

1 Rulon S. Wells comenta este problema en «De Saussure's System of Linguistics» (citado por Lane, 1970: 27). 
Des concepts et des thèmes contenus dans le Cours de Linguistique Générale ont été utilisés au centre de différentes directions de recherche. Se réclament en effet du Cours la sociolinguistique avec Meillet et Sommerfeit, la stylistique genevoise avec Bally, la linguistique psychologique avec Sechehaye, les fonctionnalistes comme Frei et Martinet, les institutionalistes italiens comme Devoto et Nencioni, les phonologues et structuralistes pragois comme Karcevskij, Trubeckoj et Jakobson, la linguistique mathématique avec Mandelbrot et Herdan, la sémantique avec Ullmann, Prieto, Trier, Lyons, la psycholinguistique avec Bresson et Osgood, les historicistes comme Pagliaro et Coseriu; et encore Bloomfield (mais pas ses disciples), Hjelmslev et son école glossématique, Chomsky (plus que ses partisans) (De Mauro, en Saussure, 1916/1972: iv).

Roland Barthes - nómada intelectual, que con el tiempo abanderaría la revista posestructuralista Tel Quel_- llegaría a protestar: el Estructuralismo no es una escuela, ni siquiera un movimiento; la mayoría de los autores etiquetados como estructuralistas no tienen conciencia de hallarse unidos por ninguna solidaridad doctrinal o compromiso común (Barthes, 1964).

¿Cierto? Probablemente no existió una conciencia sino varias (el propio Barthes es muestra de ello); puede decirse incluso que fraguó una especie de inconsciente colectivo que no compartía un mismo dominio, ni siquiera inter- o transdisciplinar, aunque sí sustentaba un común paradigma: el sistémico. Desde él cualquier objeto era abordado como expresión significativa de una totalidad orgánica de interrelaciones, estructurada según códigos socioculturalmente establecidos.

Más que un grupo definido, el Estructuralismo pergeñó un tipo de actividad investigadora cuya finalidad era crear un modelo del mundo para poner de manifiesto sus cualidades significantes. En este sentido, el concepto de estructura no suponía más que un «simulacro».

La actividad estructuralista exigía tanto la disección como la articulación. Cada objeto era fragmentado en sus elementos integrantes, delimitados por sus relaciones de posición/oposición con los demás elementos del sistema, de modo que la modificación de cualquier elemento afectaría a todos los demás y alteraría el conjunto, lo cual implicaba simultáneamente similitud y diferencia. Una vez diseccionado, el objeto era rearticulado, a través de ciertas reglas de asociación, para descubrir en él características recurrentes y formas estables que dieran razón de tales recurrencias. Los simulacros así construidos generaron una nueva categoría de objetos - ni reales ni puramente racionales, sino funcionales - que revelaban la mediación humana en los pro- 
cesos de construcción de sentido, pues el objetivo del Estructuralismo no era asignar significados a los objetos, sino descubrir cómo se construyen.

Esta lectura aproxima el Estructuralismo al Constructivismo, a la Cibernética, a la Teoría General de Sistemas y derivadamente a

[...] various modern sciences: control theory, computer science, information theory, automata theory, artificial intelligence and artificial neural networks, cognitive science, computer modeling and simulation science, dynamical systems, and artificial life. Many concepts central to these fields, such as complexity, self-organization, self-reproduction, autonomy, networks, connectionism, and adaptation, were first explored by cyberneticians during the 1940's and 1950's (Heylighen \& Joslin, 2001: 3).

\section{LA CUESTIÓN DEL INMANENTISMO}

Entre los rasgos que caracterizan tópicamente el Estructuralismo, destaca uno que condensa todos los demás y ha sido objeto primordial de la crítica: el inmanentismo. Aquí radica el supuesto desinterés de los discípulos de Saussure por la pragmática comunicativa, con todos sus accidentes y transgresiones normativas, con su evolución temporal y su materialidad significante. Ahora bien, ¿hasta qué punto es justa esta caracterización?

Es cierto que el lingüista suizo delimita claramente el ámbito de la lengua (langue) y el del habla (parole), centrando en el primero el interés de la Lingüística. Sin embargo, mantiene una concepción de la lengua que podría denominarse psicosociológica (Thibault, en Bouissac, 1998: 557), pues la presenta como una institución social que implica un acervo cultural (Saussure, 1916/1972: 32 y 33, cap. III, § 3).

Respecto a la metodología sincrónica en contraste con el diacronismo habitual en la Lingüística de su época, el Cours no se halla exento de ambivalencias: aunque se ocupa de las estructuras actuales del sistema lingüístico desde una perspectiva no evolutiva, reconoce su dimensión histórica. Precisamente por ello, la Escuela de Praga - integrada por discípulos de Saussure- muy pronto problematizó la distinción entre enfoques sincrónicos y diacrónicos.

En torno a esta Escuela - y a pesar de sus manifiestas divergencias con Trubetzkoy y Jakobson - completa su formación Louis Hjelmslev (18991965). Tres años después de participar junto con Viggo Brøndal en el I 
Congreso Internacional de Lingüística, celebrado en Ginebra en 1928, Hjelmslev funda el Círculo Lingüístico de Copenhague. Si bien asienta la «ortodoxia» del cientificismo estructuralista, su obra apunta ciertas cuestiones interesantes a las que habitualmente no se ha prestado atención.

El primer trabajo de Hjelmslev, elaborado en París -Principes de grammaire générale (1928)-, es una teoría sobre los fundamentos de la lengua concebida como sistema estructurado sobre bases inductivas. Debido a su enfoque excesivamente teorético, la investigación no fue aceptada como tesis doctoral y hubo de ser reemplazada por un análisis fonológico historicista, Études Baltiques (1932). A partir de entonces toda su labor académica estará marcada por una clara opción a favor de la metodología sincrónica y la fundamentación teorética ${ }^{2}$ que, sin embargo, se reconoce también deudora de la historia y busca la aplicabilidad empírica ${ }^{3}$, lo cual entreabre el camino hacia una ulterior síntesis:

Si l'on peut montrer que les changements linguistiques (...sont dûs) à une prédisposition au changement dans le système du langage lui-même, (...) alors la linguistique comparée aura réussi à établir une explication linguistique générale, selon laquelle les changements linguistiques sont dûs aux états linguistiques, et où le particulier est dû au général. La linguistique d'aujourd'hui approche, et atteindra probablement une synthèse de cette nature (Hjelmslev, 1971: 28).

Esta óptica se manifiesta, por ejemplo, en las ediciones de la obra de Rasmus Rask (1932, 1933, 1935), que Hjelmslev considera orientada hacia una tipología general de las estructuras lingüísticas como base de los estudios histórico-comparativos, oponiéndose así a la interpretación común en su tiempo que presentaba a Rask simplemente como lingüista histórico ${ }^{4}$.

A partir de 1935 Hjelmslev empieza a confeccionar la denominada «glosemática» en colaboración con H. J. Uldall. La glosemática arranca de la preocupación moderna sobre el atraso de las humanidades frente al progreso de las ciencias empíricas y propone como solución un álgebra de las funciones

\footnotetext{
2 «A theory, in our sense, is in itself independent of any experience. In itself, it says nothing at all about the possibility of its application and relation to empirical data. It includes no existence postulate» (Hjelmslev, 1943/1963: 14). Hjelmslev dice que — en este sentido— su teoría es arbitraria.

3 En consonancia con lo declarado en la cita anterior, Hjelmslev sostiene que su teoría busca arbitrariamente la adecuación a los datos empíricos y la aplicabilidad a todos los fenómenos de una misma clase (Hjelmslev, 1943/1963: 14-16).

4 http://mails.fju.edu.tw/ phono/cope.htm (consultado en noviembre de 2003).
} 
lingüísticas que aspira a plasmar su estructura y es potencialmente aplicable a cualquier otra estructura semiótica, en una línea inmanentista: "It may then be projected on the 'reality' outside language (...), so that language as the central point of reference remains the chief object» (Hjelmslev, 1943/1963: 8).

Todo ello requiere un empiricismo no basado en la inducción, sino en la deducción (Hjelmslev, 1943/1963: 12-13), la autoconsistencia, la exhaustividad, la simplicidad, la economía procedimental y la generalización descriptiva (Hjelmslev \& Uldall, 1957: 20 y ss.). De este modo es posible identificar los glosemas (Hjelmslev, 1943/1963: 135, §65), las formas mínimas o invariantes irreducibles de cualquier paradigma, y las leyes que rigen su articulación.

Como ya hemos apuntado, el primordial objetivo de Hjelmslev es ofrecer un tratamiento estrictamente lingüístico de la lengua (Hjelmslev, 1970: 4-5) esto es, un tratamiento inmanente. Para ello considera preciso que su teoría sea «as unmetaphysical as possible» (Hjelmslev, 1943/1963: 20) y que se centre en la dimensión formal y funcional-estructural de los fenómenos, en el sistema que subyace bajo sus manifestaciones superficiales y cambiantes, como condición de posibilidad (Hjelmslev, 1943/1963: 10). Así, «the process can be analyzed (...) into a limited number of elements recurring in various combinations (...) to set up a general and exhaustive calculus of the possible combinations» (Hjelmslev, 1943/1963: 9); ese calculus es la glosemática. Gracias a ella puede persistir la investigación de lo histórico y lo contingente, pero desde una nueva dimensión:

[...it] should rise above the level of mere primitive description to that of a systematic, exact, and generalizing science, in the theory of which all events possible combinations of elements - are foreseen and the conditions for their realization established (Hjelmslev, 1943/1963: 9).

En este marco la lengua se define como un sistema de signos que permite la formación de un número potencialmente ilimitado de nuevos signos, lo cual implica un número limitado de no-signos, partes integrantes del signo que Hjelmslev denomina figuras (Hjelmslev, 1943/1963: 46). Ahora bien, es preferible hablar de función sígnica que de signo, para expresar la solidari-

5 «To grasp language not as a conglomerate of non-linguistic (e.g., physical, physiological, psychological, logical, sociological) phenomena» (Ibid: 6). 
dad que vincula el plano significante - expresión - y el plano significado - contenido - Dentro de la glosemática, la cenemática se ocupa de la expresión; la pleremática, del contenido. Ambos planos están integrados por forma, materia y substancia: lo que permanece constante en cualquier manifestación lingüística es la forma, lo que varía es la substancia (Hjelmslev, 1943/1963: 134, §51-52). La materia es la substancia semióticamente informe; la substancia es materia informada semióticamente ${ }^{6}$.

Con todo, el principal objeto de la Lingüística son los textos (Hjelmslev, 1943/1963: 16), que constituyen el proceso respecto del cual la lengua es sistema. Una vez más el énfasis recae en las funciones que lo integran, entendidas en un sentido lógico-matemático y etimológico como dependencias susceptibles de análisis (Hjelmslev, 1943/1963: 33). Por eso la glosemática vertebra de modo sistemático una serie de definiciones conceptuales interdependientes y describe todo fenómeno en términos de relaciones, correlaciones y derivaciones, lo cual permite la comparación con otros fenómenos descritos sobre las mismas bases. Así, la inmanencia abre resquicios a una subvertida trascendencia:

The smallest system is a self-sufficient totality, but no totality is isolated. [...] It is not the individual language alone that is the object of the linguist, but the whole class of languages, the members of which are connected with each other and explain and cast light on each other [...] Accordingly, we find no non-semiotics that are not components of semiotics, and, in the final instance, no object that is not illuminated from the key position of linguistic theory. Semiotic structure is revealed as a stand from which all scientific objects may be viewed.

Linguistic theory here takes up in an undreamed-of way and in undreamed-of measure the duties that it imposed on itself. In its point of departure linguistic theory was established as immanent, with constancy, system, and internal function as its sole aims, to the apparent cost of fluctuation and nuance, life and concrete physical and phenomenological reality. [...However], instead of hindering transcendence, immanence has given it a new and better basis; immanence and transcendence are joined in a higher unity on the basis of immanence. Linguistic theory is led by an inner necessity to recognize not merely the linguistic system [...] but also man and human society behind language, and all man's sphere of knowledge through language (Hjelmslev, 1943/1963: 126-127).

\footnotetext{
${ }^{6}$ Esta precisión aparece en «La Stratification du langage» (1954), en Hjelmslev (1971: 9 y 58).
} 


\section{LA TEORÍA MATEMÁTICA DE LA COMUNICACIÓN Y LA CIBERNÉTICA}

Simultáneamente a los trabajos de Hjelmslev y Uldall se desarrollan nuevas tecnologías en el campo de la información y la comunicación. Ello favorece la creación de teorías que pretenden simultáneamente la consistencia lógica y el respaldo empírico-funcional. De este modo, la visión holística y sistémica que había provocado una revolución entre los seguidores de Saussure, aparece también en autores ajenos al Estructuralismo.

The Mathematical Theory of Communication surgió como respuesta a las demandas empresariales para mejorar los sistemas de telecomunicaciones. El Bell System Technical Journal publicó relevantes trabajos científicos con esta orientación: «Certain Factors Affecting Telegraph Speed» (abril, 1924), de H. Nyquist; «Transmission of Information» (julio, 1928), de R. Hartley; o «The Mathematical Theory of Communication» (julio y octubre, 1948), elaborado por Claude Shannon, investigador en los Bell Telephone Laboratories y en el Massachusetts Institute of Technology.

En su ensayo introductorio, Warren Weaver comienza aclarando que el término «communication» será utilizado en un vasto sentido, «to include all of the procedures by which one mind (or mechanism) may affect another» (Weaver, en Shannon \& Weaver, 1949: 3) y, por consiguiente, todos los medios posibles de comunicación: habla, música, cine, teatro, etc. Así pues, la teoría se plantea en un nivel de la máxima generalidad, que atañe a problemas fundamentales sobre la transmisión de información y puede aplicarse a ámbitos tan distintos como la traducción ${ }^{7}$ o la computación, y las ciencias cognitivas $^{8}$. De este modo, se afirma el alcance paradigmático de las nuevas investigaciones.

Su formalización permitirá definir en términos unívocos y operativos las variables implicadas en todo proceso de comunicación, comenzando por la más importante: la información. Para ello se adopta un método matemático que no supone única ni principalmente un tratamiento cuantitativo, sino un tratamiento sistémico.

\footnotetext{
7 Aunque Weaver reconoce que ello también implica cuestiones semánticas, dignas de un estudio ulterior.

8 «Either one must say that such a computer thinks, or one must substantially modify the conventional implication of the verb to think» (Weaver, en Shannon \& Weaver, 1949: 25-26).
} 
Desde esta perspectiva la información no se considera ni un contenido ni una propiedad de los mensajes ${ }^{9}$, sino una medida inversamente proporcional al grado de probabilidad de un determinado ítem dentro de un contexto delimitado y en relación con otras variables, lo cual implica una equivalencia entre este concepto de información y el concepto termodinámico de entropía.

Las variables implicadas son las alternativas disponibles para confeccionar el mensaje, teniendo en cuenta las ya elegidas anteriormente y las restricciones impuestas por el código vigente en cada caso. «Information is a measure of one's freedom of choice when one selects a message» (Weaver, en Shannon \& Weaver, 1949: 9).

Si la selección se plantea únicamente entre dos alternativas diferentes, la teoría de Shannon postula arbitrariamente que el valor de la información es uno. Esta unidad de información recibe el nombre de bit (binary digit), acuñado por J. W. Tukey. Pero aunque estén implicadas más de dos posibilidades, el cálculo sigue una lógica similar. La cantidad de información crece en la medida en que las alternativas en juego posean un grado de probabilidad más semejante y cuanto mayor sea el número de alternativas. Para que el valor de la información sea un bit, todas las opciones deben ser igualmente probables y por tanto estar igualmente disponibles: la libertad de elección no ha de hallarse precondicionada por ninguna de ellas, pues en caso de existir alguna influencia, disminuye la incertidumbre y por lo tanto la cantidad de información. Ahora bien, esto es precisamente lo habitual en la práctica comunicativa: la libertad de la fuente de información para elegir entre varias alternativas a la hora de construir su mensaje está condicionada por las alternativas elegidas anteriormente, así como por las reglas del código que utiliza. Ello supone que no todas las opciones son igualmente probables, lo cual constituye un tipo de proceso estocástico denominado Markoff ${ }^{10}$.

Probablemente lo más interesante es que el concepto de información subyacente a estos planteamientos «applies not to the individual messages (...), but rather to the situation as a whole» (Weaver, en Shannon \& Weaver, 1949: 9), que se expresa en un logaritmo del número de posibilidades disponibles. Esto revela interesantes coincidencias con los planteamientos de

9 «The semantic aspects of communication are irrelevant to the engineering aspects. But this does not mean that the engineering aspects are necessarily irrelevant to the semantic aspects» (Weaver, en Shannon \& Weaver, 1949: 8).

10 Todo sistema que produce una secuencia de símbolos según ciertas probabilidades da lugar a un proceso estocástico; si esas probabilidades están condicionadas por eventos anteriores, constituyen una cadena Markoff (Shannon \& Weaver, 1949: 11). 
Saussure y sus seguidores: se trabaja con un paradigma de la máxima generalidad, que atañe a cuestiones fundamentales y puede aplicarse en muy diversos ámbitos disciplinares. En él se subraya la dimensión relacional de la información dentro de un sistema que implica una serie de alternativas disponibles, regidas por las reglas de un código:

The concept of information developed in this theory at first seems disappointing and bizarre —disappointing because it has nothing to do with meaning, and bizarre because it deals not with a single message but rather with the statistical character of a whole ensemble of messages, bizarre also because in these statistical terms the two words information and uncertainty find themselves to be partner. I think, however, that these should only be temporary reactions; and that one should say, at the end, that this analysis has so penetratingly cleared the air that one is now, perhaps for the first time, ready for a real theory of meaning. (...) The idea of utilizing the powerful body of theory concerning Markoff processes seems particularly promising for semantic studies, since this theory is specifically adapted to handle one of the most significant but difficult aspects of meaning, namely the influence of context (Weaver, en Shannon \& Weaver, 1949: 27-28).

En lo que respecta a la inmanencia, Shannon afirma que no existen criterios puramente racionales para comprobar que la interpretación de un mensaje sea correcta, dada la imposibilidad de salir del sistema para dar razón de él: de ahí que el valor de cualquier explicación metalingüística resulte siempre meramente aproximativo. Sin embargo, la dinámica comunicativa social suele conformarse con explicaciones operativas o eficaces desde una consideración pragmática. Ello supone que en la interpretación no sólo intervienen cuestiones técnicas, sino también semánticas, conductuales, psicológicas, emocionales y estéticas.

De este modo, Shannon da un paso más allá de Saussure, Hjelmslev y Uldall, en una dirección behaviorista, al relacionar estrechamente las cuestiones semánticas y las conductuales: «With any reasonably broad definition of conduct, it is clear that communication either affects conduct or is without any discernible and probable effect at all» (Weaver, en Shannon \& Weaver, 1949: 5).

Esta afirmación se halla en consonancia con las preocupaciones de la Cibernética. Norbert Wiener - matemático, filósofo e ingeniero- es el autor de Cybernetics or Control and Communication in the Animal and the Machine (1948) y The Human Use of Human Beings. Cybernetics and Society (1950). Ambas obras sostienen que una vida plenamente humana requiere 
una capacidad de control regida por una información adecuada. En este sentido, Wiener reconoce una importante similitud entre el hombre y las máquinas: «The nervous system and the automatic machine are fundamentally alike in that they are devices which make decisions on the basis of decisions they have made in the past ${ }^{11}$.

Como se sabe, el término «Cibernética» fue acuñado a partir del griego $\chi \cup \beta \varepsilon \rho \nu \tau \eta \varsigma$ (piloto o gobernador), para designar el estudio de los sistemas de control y comunicación de los organismos vivos o de las máquinas, en una aproximación simultánea a Biología y a la Ingeniería. La Cibernética analiza y explota el carácter retroalimentativo de los circuitos que canalizan los intercambios de información entre una entidad sistémica y su entorno, permitiendo su constante adaptación al medio a través de la reacción (feed-back) o la anticipación (feed-forward) de estados. Un estado es un conjunto de pautas regladas de interacción que caracterizan a los componentes de un sistema en un momento cronológicamente determinado. La transición de un estado a otro implica cambios asimismo pautados o reglados.

La aplicación de la Cibernética al conocimiento empírico ha exigido la elaboración de modelos a través de la simulación. Ello ha abierto interesantes cuestiones epistemológicas sobre la relevancia, pertinencia y adecuación de las representaciones cognitivas. De ahí, la estrecha conexión entre la Cibernética, las ciencias de la computación y las ciencias cognitivas, preocupadas por construir modelos capaces de simular los procesos inteligentes: la inteligencia artificial $^{12}$.

Además de Matemáticas, Wiener había estudiado Filosofía y se sentía autorizado a establecer analogías a primera vista sorprendentes, como la que establece entre la bomba atómica — destructora de la realidad - $\mathrm{y}$ «the digital or computer bomb, which destroys the principle of reality itself - not the actual object- and rebuilds it» ${ }^{13}$. El alcance epistemológico e incluso ontológico de esta reflexión es de tal envergadura, que Wiener apostilla: «We have modified our environment so radically that we must now modify ourselves in order to exist in this new environment $\gg^{14}$. La naturaleza humana debe ser en-

\footnotetext{
11 Wiener, citado en http://www.angelfire.com/co/1x137/wiener.html (consultado en octubre de 2003). Con esta cita, el fundador de la Cibernética anuncia lo que será la Cibernética de segundo orden.

${ }^{12}$ La concepción de la inteligencia en términos sistémicos generó una proliferación de investigaciones de orientación esencialmente práctica, predominante en los últimos años, que se aleja progresivamente de la Cibernética, más focalizada en cuestiones teoréticas.

13 Wiener, citado en http://www.angelfire.com/co/lx137/wiener.html (consultado en octubre de 2003).

14 Ibid.
} 
tendida desde nuevos parámetros: «We are not stuff that abides, but patterns that perpetuate themselves. A pattern is a message, and may be transmitted as a message» ${ }^{15}$.

Wiener afirma así que la identidad individual se parece más a la de una llama que a la de una piedra, es la individualidad de una forma, más que la de una substancia. Esta forma puede ser transmitida, modificada o duplicada, lo cual permite la extensión de los sentidos y de la capacidad humana de actuación. «[...] where man's word goes, and where his power of perception goes, to that point his control, and in a sense his physical existence, is extended $»^{16}$.

De aquí deriva una revolucionaria visión de la sociedad que anticipa los más recientes planteamientos contemporáneos: «The community extends so far as there extends an effectual transmission of information $»^{17}$. La concepción y las técnicas de comunicación son esenciales en todo sistema social: su organización es análoga a la del individuo y su dinámica manifiesta el mismo tipo de procesos retroalimentativos (Wiener, 1948/1961: 24). Gregory Bateson llegará a decir: «The essence of our message to the reader is that communication is the matrix in which all human activities are embedded. (...) Scientifically speaking, this interrelatedness is understood best in terms of systems of communication» (Ruesch, en Ruesch \& Bateson, 1951: 13).

\section{FORMALISMO Y ESTRUCTURALISMO}

Como hemos visto, las versiones matemáticas de la interdependencia entre los elementos sistémicos se desarrollan paralelamente en el Estructuralismo y en el ámbito de las tecnologías de la información y la comunicación. Entre ambas corrientes no existe una influencia directa, pero sí importantes coincidencias que revelan una común orientación paradigmática.

La deriva inmanentista - que circunscribe la investigación a unos límites prefijados, afirmando la imposibilidad de trascender los límites del sistema para dar razón de él- está presente tanto en la glosemática como en la teoría matemática de la comunicación. Sin embargo, en ambos casos deja resquicios para ulteriores y más sutiles planteamientos. Algunos representantes

\footnotetext{
15 Ibid.

16 Ibid.

17 Ibid.
} 
de la Escuela de Praga exploraron esos resquicios al mantener una concepción funcional de las estructuras lingüísticas que no sólo expresaba un conjunto de relaciones intrasistémicas, sino también su finalidad en la actividad comunicativa.

Los primeros trabajos pretendían distinguir la práctica instrumental o cotidiana de la lengua de sus usos poéticos. El Formalismo ruso caracterizó tales usos por la desautomatización de la percepción y el extrañamiento, que atraen la atención del receptor sobre el signo - o el significante- literario. Éste deja así de funcionar como mero vehículo del significado y adquiere un valor propio.

Tal caracterización generó importantes divergencias en torno al formalismo. Puesto que los materiales de la expresión y las técnicas utilizadas para trabajar con ellos formaban parte esencial de los intereses del grupo, la noción de «forma» en contraste con la noción de «contenido» parecía inapropiada para denominar el núcleo de las nuevas investigaciones literarias.

Como hemos comentado anteriormente, las teorías de Hjelmslev contribuyeron desde fuera al debate aportando una consideración más compleja y sintética de toda función sígnica, en tanto correlación entre un plano de la expresión y un plano del contenido, cada uno de ellos articulado formal y material o substancialmente.

Pero eso no era suficiente para algunos autores. Jan Mukařovský —especialista en Poética, Estética y Epistemología del arte- quiso ir más allá, distanciándose tanto de la metodología propugnada por L. Hjelmslev como de ciertas propuestas de V. Šklovskij ${ }^{18}$ hasta invertir su máxima: «En la obra de arte todo es forma», y reemplazarla por esta otra: «En la obra de arte todo es contenido» ${ }^{19}$.

Mukařovský es habitualmente considerado un autor estructuralista: miembro fundador del Círculo de Praga en 1926 y profundo conocedor del Formalismo tanto ruso como checo, principalmente a través del magisterio de I. Tynjanov y R. Jakobson. Sin embargo, su obra revela una apertura a la complejidad que anticipa todos sus desarrollos y correcciones posteriores. Su visión es muy original y crítica, condensa lo mejor de las mencionadas escuelas pero también cuestiona sus límites, asociándose en algún momento al

${ }_{18}$ Mukar̆ovský: «A note on the Czech Translation of S̆klovskij's Theory of Prose», en Mukar̆ovský (1977: 134-142).

${ }^{19}$ Citado por Bouissac (1998: 519). 
Marxismo. «Mukar̆ovský's structuralist aesthetics must therefore be seen as a process, a way of posing questions rather than a closed system» (Steiner, en Mukařovský, 1978: x).

En lo que concierne al concepto de «estructura» y su análisis desde una perspectiva sincrónica, Mukar̆ovský puntualiza lo siguiente:

Structure is usually defined as a whole, the parts of which acquire a special character by entering it. (...) However, this definition is too broad. (...) We designate interrelations among the components — relations dynamic in their very essence - as the specific property of structure in art. According to our conception we can consider as a structure only such a set of elements, the internal equilibrium of which is constantly disturbed and restored anew and the unity of which thus appears to us as a set of dialectic contradictions. That which endures is only the identity of a structure in the course of time, whereas its internal composition - the correlation of its components-changes continuously (Mukařovský: «On Structuralism», en Mukařovský, 1978: 3-4).

La metodología formalista también es asumida sólo dentro de ciertos límites, críticamente:

Thus structuralism does not limit literary history only to an analysis of 'form', nor does it clash at all with sociological studies of literature. It does not restrict the scope of the material or the wealth of problems, but it insists on the postulate that scholarly study not regard its material as a static and atomized chaos of phenomena but that it conceive every phenomenon as a resultant and source of dynamic impulses and the whole as a complex interplay of forces (Mukařovský: «A note on the Czech Translation of Sklovskij's Theory of Prose», en Mukařovský, 1977: 141).

Las reflexiones «Sobre el lenguaje poético» (O jazyce básnickém), presentadas por Mukařovský en el I Congreso Internacional de Eslavistas en Praga (1929), anticipan su novedosa metodología, que atiende simultáneamente a los aspectos funcionales, formales y materiales, estáticos y dinámicos, textuales y contextuales de la poesía. Ésta no se distingue por su carácter ornamental, ni por su belleza. Tampoco por su emotividad, su concreción o alguna especial propiedad:

No single property characterizes poetic language permanently and generally. Poetic language is permanently characterized only by its function; however, function is not a property but a mode of utilizing the properties of a given phenomenon (Mukařovský: «On Poetic Language», en Mukařovský, 1977: 3-4). 
El modo poético de utilizar el lenguaje «concentrates attention on the linguistic sign itself $\mathrm{f}^{20}$-hence it is exactly the opposite of a practical orientation toward a goal which in language is communication» (Mukařovský, 1977: 4). Sin embargo, no conviene magnificar esta oposición: dado que toda obra de arte consta de una compleja interrelación de elementos sistemáticamente organizados, lo más característico de ella es su carácter polifuncional. Las funciones prácticas o instrumentales - así como las funciones representativa, expresiva y apelativa analizadas por K. Bühler - conviven con la función estética de tal modo que ésta sólo llega a ser dominante en determinados contextos sociopragmáticos:

There is a constant struggle and a constant tension between self-orientation and communication so that poetic language, though it stands in opposition to the other functional languages in its self-orientation, is not cut off from them by an insurmountable boundary (Mukařovský: «On Poetic Language», en Mukařovský, 1977: 6).

El arte carece, pues, de fronteras nítidas. La estética impregna cualquier actividad humana y su presencia, lejos de obstaculizar el ejercicio de otras funciones, las favorece.

If other 'practical' functions compete with one another when they are juxtaposed and strive to dominate one another, exhibiting a tendency toward functional specialization (toward monofunctionality, which culminates in the machine), then it is precisely because of the aesthetic function that art tends toward as rich and as many-sided a polyfunctionality as possible, without, at the same time, preventing the work of art from having a social effect (Mukařovský: «On Structuralism», en Mukařovský, 1978: 12).

La función estética permite evitar el unilateralismo de la especialización, que empobrece la relación del hombre con el mundo. Al carecer de un contenido inequívoco, las obras de arte son transparentes y revelan tanto la naturaleza del signo como la posición del hombre respecto a él.

Precisely because of its aesthetic 'self-orientation' poetic language is more suited than other functional languages for constantly reviving man's attitude toward language and the relation of language to reality, for constantly revealing in new ways the internal organization of the linguistic sign, and for sho-

${ }^{20}$ Aunque no debe confundirse con la función metalingüística de la lógica. 
wing new possibilities of its use (Mukařovský: «On Poetic Language», en Mukařovský, 1977: 6).

En último término, ello conlleva una orientación hacia la realidad. «It is clear that the aesthetic function (...) is one of the most important factors creating man's attitude toward reality» (Mukařovský, 1978: 12). Ahora bien, esto no quiere decir que las obras de arte posean un inmediato valor documental, ni que constituyan representaciones miméticas:

The true essence of the subject (of the artistic work) is a unity of meaning and not a passive copy of reality, even in the case of a 'realistic' or 'naturalistic' work (Mukar̆ovský: «Art as Semiotic Fact», en Matejka \& Titunik, 1976: 7).

It is the entire structure which functions as the signification of a work of art [...]. The subject of a work simply plays the role of an axis of crystallization with respect to that signification which, otherwise, would remain vague (Mukařovský: «Art as Semiotic Fact», en Matejka \& Titunik, 1976: 6-7).

De esta manera, Mukařovský se plantea explícitamente la cuestión del sentido y reflexiona sobre «El arte como hecho semiótico» en el Congreso Internacional de Filosofía de París de 1934. Uno de los primeros obstáculos que esta investigación despeja es el psicologismo, en consonancia con Saussure y el Estructuralismo: «The work of art cannot be identified (...) with its author's state of mind or with any of the states of mind which it evokes in perceiving subjects» (Mukařovský: «Art as Semiotic Fact», en Matejka \& Titunik, 1976: 3). Tampoco debe confundirse la obra en cuanto artefacto con la obra en cuanto objeto estético. El artefacto es el vehículo significante y perceptible de una significación registrada en la conciencia colectiva; sólo ésta constituye el objeto estético como tal. A ello hay que añadir el significado de la obra, que es el contexto de todos los fenómenos sociales - filosóficos, religiosos, políticos, económicos...- de donde procede: de ahí su carácter autónomo ${ }^{21}$ y autotélico ${ }^{22}$. Estos tres aspectos — significante, significación y significado- articulan la obra como hecho semiótico:

Only the semiotic point of view allows theorists to recognize the autonomous existence and essential dynamism of artistic structure and to understand evolution of art as an immanent process but one in constant dialectical rela-

21 Mukar̆ovský: «Art as Semiotic Fact», en Matejka \& Titunik (1976: 3-9, esp. p. 5).

22 «The object functions only vis-à-vis itself» (Mukařovský: «Problémy estetické hodnoty», p. 17, en Mukařovský, 1977: xxiii). 
tionship with the evolution of other domains of culture (Mukařovský: «Art as Semiotic Fact», en Matejka \& Titunik, 1976: 8).

La conciencia social genera el arte al determinar los usos lingüísticos y al identificar aquellos que se vuelven sobre sí mismos, transparentando tal conciencia. Como ya se ha dicho, ninguna obra de arte se define simplemente por una singular interrelación de elementos, ni por el reflejo de una psicología individual, sino por un consenso colectivo que la convierte en hecho social:

For the work once finished ceases to be a mere expression of its author's psychic state and becomes a sign, i.e., a sui generis social fact which serves supra-individual communication, severed from the subjective psychology of its author ${ }^{23}$.

Este proceso de objetivación involucra también al receptor, quien percibe la obra a través de las mediaciones sociales:

Via the intermediary of the core belonging to the collective consciousness, the subjective constituents of the perceiver's mental state acquire an objective semiotic character (Mukařovský: «Art as Semiotic Fact», en Matejka \& Titunik, 1976: 4).

Mukařovský se aparta así de la simplificación que opone sin matices objetivismo y subjetivismo en el ámbito estético. La belleza no es una Idea metafísica ni una propiedad de las cosas, como muestra la variabilidad de los juicios estéticos. Pero tampoco es totalmente subjetiva. La dimensión perceptible y significante de la obra de arte contiene cierta información que no depende de ninguna subjetividad, sino de sus propiedades formales. Por otra parte, existen normas estéticas objetivas en la conciencia colectiva, que varían históricamente pero que son fijas y vinculantes desde una perspectiva sincrónica. Debido a ello, la obra artística carece de estructura unívoca: es ambigua, susceptible de distintas actualizaciones en distintos momentos.

Con estas consideraciones Mukařovský postula un particular tipo de inmanentismo, que se abre a consideraciones contextuales o diacrónicas. «Mukar̆ovský shows how the immanent development of art is affected by its

${ }^{23}$ Mukar̆ovský: «The Artist's Personality in the Mirror of the Work: Some Critical Remarks on the Theory and Practice of the Study of Art», Cestami, p. 145 (citado en Mukařovský, 1978: xvi). 
place in the hierarchy of cultural structures» (Steiner, en Mukařovský, 1978: xxi). Todos los códigos funcionales coexisten, se entrelazan y luchan por la dominación en esa estructura última que es la cultura de una sociedad.

La culminación de estas reflexiones es la obra Función estética, norma y valor como hechos sociales (1936). En ella Mukar̆ovský señala que el valor estético - frente a otro tipo de valores - no nace de la obediencia a la norma, sino viceversa, hasta el punto de que frecuentemente la norma se manifiesta precisamente al ser violada. La norma artística es un principio regulatorio, difícilmente codificable e inestable, más que un rígido a priori. Debido a su valor, cada obra artística es única e irrepetible, una totalidad cerrada, donde la función estética domina - pero a la vez convive- con otras funciones:

If, at this point, we ask ourselves where aesthetic value is, we find that it has dissolved into the individual extra-aesthetic values and is nothing but a general designation for the dynamic totality of their interrelations (Mukar̆ovský: «Estetická funkce», p. 51, en Mukařovský, 1977: xxv).

El carácter social de tal designación podría favorecer una deriva relativista que Mukařovský desea evitar. Para ello atiende no ya a los aspectos sistémicos cuanto a sus condiciones de posibilidad: pasa de la «lengua» al «lenguaje», de los códigos culturales a los universales de la cultura.

Además, fuertemente influido por la Fenomenología, rescata el papel del sujeto, hasta ahora difuminado en la impersonalidad de la conciencia colectiva y subordinado a sus estructuras. El actor social es tanto creador como perceptor, partícipe en el proceso estético, determinado por ciertas constantes biológicas y antropológicas, libre frente a los sistemas culturales, una fuente de accidentalidad frente a todo supuesto determinismo. La combinación de las perspectivas universalista y humanista soslaya todo posible subjetivismo:

What is it that makes [some] phenomena universal objects of aesthetic experience? [...] The close affinity between their organization and the constitutive principles stemming from man's anthropological constants (Steiner, en Mukar̆ovský, 1978: xxxi).

El significado de la obra «arises in a process in which the perceiving subject brings together all the partial meanings within the work. Moreover, in art, it is not this result which is important but the process itself» (Steiner, en Mukařovský, 1978: xxxii). 
Con estas reflexiones, Mukařovský subraya la importancia de la actividad perceptiva en la constitución de la obra estética, anticipándose a H. R. Jauss y la Escuela de Constanza.

\section{BALANCE FINAL}

En su tiempo, Roland Barthes comprendió —no sin cierta ironía y sutileza intelectual- que el marketing académico pronto desecharía el Estructuralismo:

Le structuralisme ne retire pas au monde l' histoire: il cherche à lier à l' histoire, non seulement des contenus (...), mais aussi des formes, non seulement le matériel, mais aussi l'intelligible, non seulement l'idéologique, mais aussi l'esthétique. Et précisément, parce que toute pensée sur l'intelligible historique est aussi participation à cet intelligible, il importe peu, sans doute, à l' homme structural de durer: il sait que le structuralisme est lui aussi une certaine forme du monde, qui changera avec le monde; et de même qu'il éprouve sa validité (mais non sa verité) dans son pouvoir à parler les anciens langages du monde d'une manière nouvelle, de même il sait qu' il suffira que surgisse de l'histoire un nouveau langage qui le parle à son tour, pour que sa tâche soit terminée $e^{24}$.

Sin embargo, este artículo nos ha permitido explorar algunas contribuciones «estructuralistas» que cuestionan el relato canónico transmitido por la comunidad intelectual. También hemos descubierto una importante continuidad entre el Estructuralismo y la Cibernética, basada en el paradigma sistémico.

Urge pues, rehabilitar el Estructuralismo, no ya para recuperarlo o para rescatarlo del tiempo, sino para entendernos a nosotros mismos, hoy y ahora, dado que sus aportaciones se integran en la misma corriente de pensamiento que actualmente nutre la vanguardia de nuestras investigaciones sociales.

${ }^{24}$ Barthes, 1964: 219-220 (fragmento de «Lettres Nouvelles», 1963). 


\section{REFERENCIAS BIBLIOGRÁFICAS}

BARThes, R. (1964). Essais critiques. Paris: Ed. Du Seuil.

Bouissac, P. (1998). Encyclopedia of Semiotics. Oxford \& New York: Oxford University Press.

Heylighen, F., \& Joslyn, C. (2001). «Cybernetics and Second-Order Cybernetics». En R. A. Meyers (ed.), Encyclopedia of Physical Science \& Technology. New York: Academic Press. Publicado en http://pespmcl. vub.ac.be/Papers/Cybernetics-EPST.pdf.

HJelmslev, L. (1943/1963). Prolegomena to a Theory of Language. Madison: University of Wisconsin Press.

- (1970). Language: An Introduction. Madison-Milwaukee-London: University of Wisconsin Press.

— (1971). Essais linguistiques. Paris: Éd. de Minuit.

HJelmslev, L., \& Uldall, H. J. (1957). Outline of Glossematics. A Study in the Methodology of the Humanities with Special Reference to Linguistics. Copenhague: Nordisk Sprog- og Kulturforlag.

LANE, M. (ed.) (1970). Introduction to Structuralism. New York: Basic Books.

MatejKa, L., \& TituniK, I. R. (eds.) (1976). Semiotics of Art. Prague School Contributions. Cambridge, Mass. \& London: The MIT Press.

MukařovskÝ, Jan (1977). The Word and Verbal Art. Selected Essays by Jan Mukărovský. Translated and edited by John Burbank and Peter Steiner. New Haven \& London: Yale University Press.

- (1978). Structure, Sign, and Function. Selected Essays by Jan Mukařrovský. Translated and edited by John Burbank and Peter Steiner. New Haven \& London: Yale University Press.

RIEBER, R. W. (ed.) (1989). The Individual, Communication, and Society. Essays in Memory of Gregory Bateson. Cambridge: Cambridge University Press.

Ruesch, J., \& BAteson, G. (1951). Communication: The Social Matrix of Psychiatry. New York: W.W. Norton \& Co.

Saussure, F. DE (1916/1972). Cours de Linguistique Générale. Charles Bally, Albert Sechehaye \& Albert Riedlinger (eds.); Tullio de Mauro (ed. crítica). Paris: Payot. 
Shannon, C. E., \& WeaVer, W. (1949). The Mathematical Theory of Communication. Urbana-Chicago-London: University of Illinois Press.

Toulmin, S. (1964). «The Importance of Norbert Wiener». The New York Review of Books, vol. 3, n. 3, September 24th. Publicado en http://www.nybooks.com/articles/13224.

VV. AA. (1949). Recherches structurales. Interventions dans le débat glossématique. Copenhague: Nordisk Sprog — og Kulturforlag.

WIENER, N. (1948/1961). Cybernetics or Control and Communication in the Animal and the Machine. Cambridge, Mass.: The MIT Press.

- (1950). The Human Use of Human Beings. Cybernetics and Society. Boston: Houghton Mifflin Co.

\section{Fuentes digitales}

http://mails.fju.edu.tw/ phono/cope.htm

http://www.angelfire.com/co/1x137/wiener.html

http://pespmcl.vub.ac.be/Papers/Cybernetics-EPST.pdf

http://www.nybooks.com/articles/13224 